\title{
Análise ergonômica do trabalho em unidades de beneficiamento de tomates de mesa: movimentação manual de cargas
}

\author{
Ergonomic work analyses method in fresh tomato packing houses: manual material handling
}

\author{
Ivan Augusto Vall Ribeiro ${ }^{I^{*}}$ Mauro José Andrade Tereso ${ }^{I}$ Roberto Funes Abrahão
}

RESUMO

A movimentação manual de cargas (MMC) nas unidades de beneficiamento de tomates (UB) é a atividade laboral mais freqüente nas etapas do processo de beneficiamento. $O$ objetivo deste estudo foi analisar, sob a ótica da ergonomia, as tarefas de MMC em seis UB de tomate de mesa do Estado de São Paulo. Foi aplicado o método da análise ergonômica do trabalho (AET) para conhecimento dos problemas associados à carga laboral. Nos postos de trabalho onde a MMC foi predominante, aplicou-se o método da equação do National Institute for Occupational Safety and Health (NIOSH) para determinar o Limite de Peso Recomendado e o método do Diagrama das Áreas Dolorosas para identificar as áreas do corpo dos trabalhadores que apresentam desconforto. Em todas os casos estudados, o peso real da carga movimentada manualmente superou o limite de peso recomendado pelo NIOSH.

Palavras-chave: movimentação manual de cargas, unidade de beneficiamento, diagrama das áreas dolorosas, equação niosh.

\section{ABSTRACT}

The manual material handling $(\mathrm{MMH})$ is the most frequent labor activity in tomato packing houses. The main goal of this paper was to analyze, under an ergonomic point of view, the MMH tasks in six fresh tomato packing houses. The ergonomic work analyses method was applied for an overall approach of work activities. The NIOSH equation was applied to calculate the recommended weight limit and a Body Discomfort Diagram was used to identify the painful body workers areas. In all cases, the actual load overcame the recommended weight limits by NIOSH.

Key words: manual material handling, packing house, painful diagram area, NIOSH equation.

\section{INTRODUÇÃO}

A produção agropecuária brasileira é extremamente importante para a economia do Brasil, correspondendo a $40 \%$ do produto interno bruto (PIB). A tomaticultura representa um dos maiores mercados agrícolas brasileiros, ocupando uma área plantada de 58.000 hectares dos quais 40.000 hectares são de tomate fresco e 18.000 hectares são de tomate destinado ao processamento. A produção anual é de aproximadamente três milhões de toneladas por ano, sendo 1 milhão e 800 mil toneladas de tomate de mesa que, em sua maioria, passa pelo processo de beneficiamento. Essa produção é realizada por aproximadamente 10.000 unidades produtoras, envolvendo mais de 60.000 famílias de trabalhadores, o que pode significar mais de 200.000 empregos diretos. A maior parte da produção de tomate concentra-se nos Estados de Goiás, São Paulo e Minas Gerais, totalizando cerca de 65\% da produção nacional (PEIXOTO, 2003).

Segundo FERREIRA (2003), o tomate de mesa há algum tempo era classificado no campo. Atualmente, o processo de seleção e classificação de tomate é feito predominantemente em Unidades de Beneficiamento (UB), que dispõem de diferentes tecnologias, formas de organização do trabalho e graus de mecanização. As operações básicas mais importantes do manuseio pós-colheita nas UB são: 0 descarregamento; a armazenagem; a limpeza; a lavagem; a secagem; a seleção; a classificação; a embalagem; a unitização ou paletização; a armazenagem (refrigerada,

IFaculdade de Engenharia Agrícola, Área de Planejamento e Desenvolvimento Rural Sustentável, Universidade Estadual de Campinas (UNICAMP). Av. Candido Rondon, 501, Barão Geraldo, Cidade Universitária Zeferino Vaz, CP 6011, 13083-875, Campinas, SP, Brasil. E-mail: tibuja@uol.com.br.*Autor para correspondência. 
em atmosfera controlada ou modificada); o carregamento; e o transporte.

Este trabalho abordou os aspectos relativos à Movimentação Manual de Cargas (MMC) em UB de Tomates. Dentre as atividades laborais que compõem o processo de beneficiamento de tomates, a MMC é caracterizada por tarefas com ritmos intensos, associadas a períodos de longa duração, que expõem os trabalhadores a riscos de lesão do sistema osteomuscular.

O processo de beneficiamento é composto por várias etapas. Em algumas delas, a MMC apresentase de forma intensa, seja pelo peso das cargas movimentadas, seja pelo ritmo (freqüência de levantamentos) ou ainda pela associação de dois ou mais fatores. Nas UB de tomates, existe uma grande variedade de tarefas que envolvem a MMC. Essas tarefas têm características diferentes e envolvem vários fatores, exigindo que os trabalhadores adotem posturas corporais e freqüências de levantamentos e abaixamentos variadas para cada uma delas.

A MMC constitui-se numa das principais causas de distúrbios osteomusculares que acometem os trabalhadores, principalmente quando é associada a pesos elevados e a esforços repetitivos por longos períodos. Outros fatores, como empurrar, puxar, levantar e abaixar cargas, além de posturas corporais inadequadas, estão diretamente relacionados com o surgimento desses distúrbios.

FREIVALDS et al. (1998) avaliaram que o levantamento de cargas pesadas causa um alto estresse no sistema osteomuscular dos trabalhadores, resultando em $25 \%$ de todas as horas não-trabalhadas no setor industrial. Além disso, para GRANDJEAN (1998), a movimentação manual de cargas deve ser considerada como trabalho pesado e tem como principal conseqüência os distúrbios da coluna vertebral e, em especial, o desgaste dos discos intervertebrais.

CHAFFIN et al. (2001) recorreram ao relatório do National Institute for Occupational Safety and Health (NIOSH) para afirmar que um terço dos trabalhadores dos Estados Unidos da América (EUA) estava envolvido em atividades de trabalho com MMC, responsáveis por $23 \%$ de todas as doenças ocupacionais, estimando um custo de US\$ 5,2 bilhões por ano. Desse relatório destacam-se os seguintes dados estatísticos: a sobrecarga foi a causa das lombalgias em mais de 60\% dos trabalhadores com queixas de dores lombares; menos de um terço dos trabalhadores afastados por acometimento de lombalgias, em função de lesões por sobrecarga, retornou ao posto de trabalho que as desencadeou; cerca de $25 \%$ de todas as lesões ocupacionais nos EUA são decorrentes de atividades com sobrecarga; o levantamento de cargas estava envolvido com aproximadamente $70 \%$ das queixas de lesões por sobrecarga.

A MMC em unidades de beneficiamento brasileiras pode ser mais ou menos intensa conforme $o$ nível de mecanização empregado para movimentar as cargas. Existem UB que, em algumas etapas do processo, possuem dispositivos que dispensam o uso de força física, como no caso do translado de paletes pelo uso de paleteiras elétricas ou de empilhadeiras. No entanto, em algumas fases do processo (empilhamento de caixas nos paletes), o uso de força física é indispensável, pois não existe tecnologia disponível no mercado brasileiro para substituí-la.

TEIXEIRA (2004) desenvolveu um procedimento sistemático para medir as variáveis da tarefa de movimentação manual de cargas necessárias à aplicação do método NIOSH e avaliar a relação entre o índice de levantamento (IL) obtido por meio da equação e a incidência de lombalgia nos trabalhadores de seis indústrias do setor metal-mecânico e de alimentos. Foi observado que a incidência de lombalgia estava relacionada às tarefas que apresentavam condições mais desfavoráveis quanto aos deslocamentos da carga e à freqüência de levantamentos.

O NIOSH desenvolveu, em 1981, uma equação para avaliar a manipulação de cargas no trabalho. Esse instituto criou uma ferramenta para identificar os riscos de distúrbios osteomusculares associados à carga física a qual o trabalhador está submetido e recomendar um limite de peso adequado para cada tarefa em questão, de maneira que uma determinada porcentagem da população pudesse realizar a tarefa sem risco de desenvolver distúrbios osteomusculares. Em 1991, a equação foi revista e novos fatores foram introduzidos: a manipulação assimétrica de cargas, a duração da tarefa, a freqüência dos levantamentos e a qualidade da pega (WATERS et al., 1994). A equação NIOSH é baseada no conceito de que o risco de distúrbios osteomusculares aumenta com o distanciamento entre o limite de peso recomendado e o peso efetivamente manipulado. Desse modo, essa equação estipula o cálculo do índice de levantamento (IL), que é obtido pelo quociente entre o peso da carga levantada ou o peso real (PR) e o peso da carga recomendada (LPR).

Devido à relevância do tema, este estudo teve como objetivo geral analisar as tarefas de MMC nas UB de tomate de mesa visando contribuir para a prevenção das ocorrências de distúrbios 
osteomusculares. Seus objetivos específicos foram: verificar a relação entre a carga recomendada para a movimentação segura, segundo o método da equação do NIOSH, e os determinantes das tarefas que condicionam a movimentação de cargas; identificar, por meio do Diagrama das Áreas Dolorosas, as áreas do corpo dos trabalhadores que, ao final de um período de trabalho, apresentem desconforto (dores); e verificar se existe correlação com os resultados obtidos pela equação do NIOSH.

\section{MATERIAL E MÉTODOS}

Para a realização deste estudo, foram selecionadas seis UB de tomates, localizadas no Estado de São Paulo, que apresentassem diferentes características, como o número de operadores, o volume de produção, o número de linhas de clasificação, o fluxo de produção, o layout dos galpões e a organização do trabalho. A tabela 1 apresenta as características de cada UB.

Para melhor compreender o funcionamento geral de uma UB, foi aplicado o método da Análise Ergonômica do Trabalho (AET) em um estudo-piloto, o qual possibilitou compreender o fluxo da produção e o funcionamento do processo de beneficiamento, além de identificar as etapas e os postos onde a MMC se apresentava de forma predominante e de que forma era executada.

Em todas as seis UB foram selecionados os postos onde havia MMC: descarregamento, alimentação, paletização, pesagem e carregamento. Em cada um desses postos foi aplicado o método da equação do NIOSH e do Diagrama das Áreas Dolorosas, envolvendo, ao todo, 63 diferentes operadores.

Para a realização da coleta de dados, foram utilizadas: uma câmera de vídeo com tripé, que foi utilizada para o registro das atividades de MMC; uma câmera fotográfica digital para registrar os momentos de maior interesse relativos à observação sistemática; um gravador de voz digital para registrar as verbalizações dos trabalhadores; um cronômetro para determinar a freqüência das repetições; um marcador manual de repetições para auxiliar as contagens; e planilhas para as anotações das observações.

Foi necessário adaptar o momento de abordagem para entrevistar os trabalhadores. Nas UB onde os trabalhadores exerciam apenas uma tarefa, a entrevista foi realizada ao final do período de trabalho. Nas UB em que os trabalhadores executavam mais de uma tarefa, a abordagem foi realizada ao final de cada uma delas. Nos postos em que havia mais de um trabalhador, o tratamento dos dados obtidos foi realizado por sobreposição, ou seja, os resultados foram colocados em um só diagrama para a representação do posto. Assim como o método da equação do NIOSH, o método do diagrama das áreas dolorosas foi aplicado em todos os trabalhadores dos postos com MMC.

Para a aplicação da equação do NIOSH, foram observados individualmente todos os postos selecionados para o estudo. As variáveis que compõem a equação foram aferidas uma a uma para todos os trabalhadores de cada um dos postos durante uma jornada de trabalho. O período de observação e a coleta de dados para cada trabalhador foram de, no mínimo, 60 minutos. As aferições não interferiram no ritmo de trabalho, pois em nenhum caso houve necessidade de interrupção das tarefas.

A equação do NIOSH que calcula o limite de peso recomendado é a seguinte:

$$
\begin{aligned}
& \mathrm{LPR}=23 \bullet\left(\frac{25}{H}\right) \bullet[1-(0.003 \bullet|V-75|)] \bullet \\
& {\left[0.82+\left(\frac{4.5}{D}\right)\right] \bullet[1-(0.0032 \bullet A)] \bullet F \bullet C}
\end{aligned}
$$

A equação do NIOSH para o levantamento de cargas determina o limite de peso recomendado (LPR) a partir de seis fatores de redução da constante de carga. Os coeficientes, que variam entre 0 e 1 , levam em conta a distância horizontal entre a carga e o operador (H); a distância vertical (V) da origem da carga; o deslocamento vertical (D) entre a origem e o destino da carga; o ângulo de assimetria (A) medido a partir do plano sagital; a freqüência média de levantamentos (F) e a qualidade da pega (C). O valor da constante de

Tabela 1 - Características das Unidades de Beneficiamento

\begin{tabular}{lccccc}
\hline Parâmetro característica & UB1 & UB2 & UB3 & UB4 & UB5 \\
\hline № de operadores em MMC & 09 & 11 & 12 & 09 & 08 \\
Produção (caixas/mês) & 8.000 & 163.800 & 458.640 & 4.800 & 131.040 \\
№ de linhas de classificação & 03 & 06 & 06 & 03 & 04 \\
Galpão & Adaptado & Adaptado & Projetado & Adaptado & $\begin{array}{c}\text { Parcialmente } \\
\text { Projetado }\end{array}$ \\
Layout das linhas de beneficiamento & Projetado \\
& Linha Reta & Linha Reta & Linha Reta & Em L & Em E \\
\hline
\end{tabular}

Ciência Rural, v.39, n.4, jul, 2009. 
carga foi estabelecido para o método NIOSH em 23kg por meio de critérios biomecânicos, psicofísicos e fisiológicos. Esse valor refere-se à movimentação de carga no plano sagital a uma altura de $75 \mathrm{~cm}$ do solo, para um deslocamento vertical de $25 \mathrm{~cm}$, para cima ou para baixo e com a carga a uma distancia máxima de 25cm do corpo do operador (IIDA, 2005). O caráter multiplicativo da equação faz com que o valor limite de peso recomendado vá diminuindo à medida que se afaste das condições ótimas.

Em conformidade com o Ministério do Trabalho e Emprego (BRASIL, 2002), foram consideradas três zonas de risco segundo os valores do índice de levantamento obtidos para a tarefa. O risco é considerado limitado quando o índice de levantamento (IL) é inferior a 1. Nesse caso, a maioria dos trabalhadores que realizam esse tipo de tarefa não deveria desenvolver distúrbios. Quando o risco é moderado $(1<\mathrm{IL}<3)$, alguns trabalhadores podem adoecer ou sofrer lesões se realizarem essas tarefas. Nesse caso, as tarefas devem ser redesenhadas ou atribuídas apenas a trabalhadores selecionados que serão submetidos a controle. No caso de risco elevado (IL $\geq 3$ ), a tarefa é inaceitável do ponto de vista ergonômico e deve ser modificada.

O Diagrama das Áreas Dolorosas também foi aplicado aos mesmos operadores. Trata-se de um método subjetivo, composto por um questionário e um diagrama do corpo humano, dividido em 24 áreas, em que os trabalhadores apontaram para as áreas do corpo em que sentiam desconforto e, por meio de uma escala, determinaram o grau desse desconforto. Com o intuito de facilitar a compreensão dos trabalhadores e agilizar a aplicação do diagrama, ficaram estabelecidas, dentro de uma escala de 0 a 7 , três faixas de avaliação de desconforto, descritas a seguir: $0-1-2=$ Nenhum desconforto até pouco desconfortável (cor branca); 3 - 4 = Desconfortável (cor amarela); Muito desconforto a extremamente desconfortável (cor vermelha).

Na análise estatística dos dados, foi utilizado o teste de Kruskal-Wallis, não-paramétrico, para verificar as diferenças significativas entre os dados ( $\mathrm{P}$ $<0,0001)$. Para as comparações múltiplas, ou seja, para os pares de grupos, realizou-se o teste de StudentNewman-Keuls (SNK), ao nível de significância de 5\%. Utilizou-se o software SAS, versão 8, para calcular os valores estatísticos.

\section{RESULTADOS E DISCUSSÃO}

No que diz respeito à movimentação manual de cargas, quanto maior o valor do Limite de Peso recomendado pela equação do NIOSH, melhor configurada está a tarefa. É importante destacar que o peso médio das caixas nos postos de descarregamento e de alimentação é de $24 \mathrm{~kg}$ e que nos postos de pesagem, paletização e carregamento de caminhões, é de $22 \mathrm{~kg}$. Como a constante de carga adotada pelo NIOSH é de 23kg, para uma configuração ideal da tarefa de movimentação manual, o peso da carga manipulada pelos operadores já é superior ou se encontra muito próximo desse limite.

A figura 1 apresenta os valores dos Limites de Pesos Recomendados (LPR) médios encontrados nas UB. O posto de descarregamento de caminhões foi o que apresentou os valores de LPR mais preocupantes, confirmando as verbalizações dos trabalhadores que o apontaram como o de maior desgaste físico. Dos seis parâmetros que compõem a equação do NIOSH, os que mais impactaram os resultados foram o período de exposição, a freqüência de levantamentos/ abaixamentos e as alturas dos deslocamentos.

A figura 2 apresenta os resultados de LPR e IL obtidos pela equação do NIOSH e do Diagrama das Áreas Dolorosas de forma agrupada. A maioria dos valores de índice de levantamento apresentou-se na zona de risco moderado $(1<\mathrm{IL}<3)$, indicando que as tarefas devem ser reorganizadas com o intuito de reduzir os riscos de lesão do sistema osteomuscular dos trabalhadores. Os relatos dos trabalhadores sobre as áreas do corpo com sintoma “desconfortável” e “extremamente desconfortável” são muito semelhantes nas seis UB, destacando-se a coluna lombar e sacral, os ombros, o pescoço, os braços e o antebraço. Os resultados obtidos pelo Diagrama das Áreas Dolorosas reforçam as verbalizações dos trabalhadores e corroboram os valores mais elevados de índice de levantamento obtidos pelo método NIOSH.

\section{CONCLUSÕES}

A MMC apresentou-se muito intensa nos diversos postos que compõem o fluxo de produção nas UB de tomate de mesa. Essa intensidade é decorrente dos fatores determinantes das tarefas de MMC, como ritmo, altura inicial das caixas, distâncias dos deslocamentos das caixas e peso das caixas movimentadas. Esses fatores estão diretamente ligados ao volume de produção, tipo de tecnologia empregada e número de trabalhadores disponível para executar as tarefas.

O processo de produção das UB emprega alta tecnologia no que diz respeito aos equipamentos (máquinas) de beneficiamento. Tais equipamentos são compostos por dispositivos com tecnologia digital (computadores, câmeras de vídeo e sensores de 


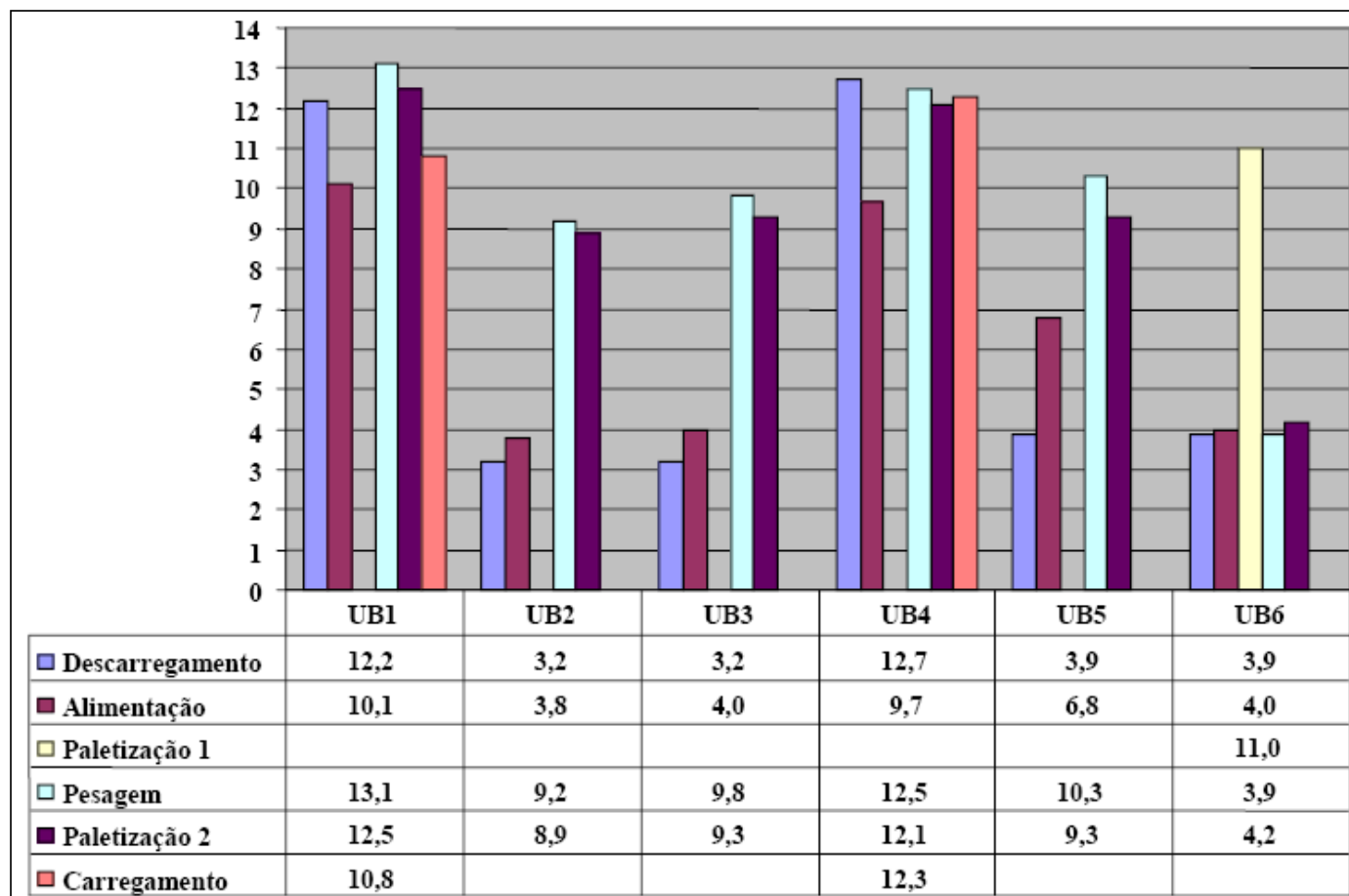

Figura 1 - Limites de pesos recomendados médios (em kg) nos postos de trabalho com movimentação manual de cargas nas Unidades de Beneficiamento

temperatura), sendo muitos importados. No entanto, nas etapas do processo em que predomina o trabalho humano, tecnologias para o auxílio da movimentação de carga foram identificadas somente no posto de carregamento de caminhões em algumas UB. Ainda assim, o dispositivo de auxílio mais comum é a paleteira mecânica, que exige grande esforço físico para sua manipulação.

A avaliação das atividades em que ocorre a movimentação manual de cargas em todas as UB de tomate estudadas, por meio do método NIOSH e do Diagrama de Áreas Doloridas, permite concluir que todos esses postos precisam ser modificados, já que os riscos são moderados ou elevados em todos os casos.

Os postos de descarregamento de caminhões e de alimentação da máquina merecem a maior atenção nas UB. Os valores dos índices de levantamento encontram-se na zona de risco moderado e de risco elevado. O Diagrama das Áreas Dolorosas também apresentou resultados que inspiram atenção. Os trabalhadores relataram extremo desconforto em áreas do corpo com grande potencial de lesões osteomusculares quando muito exigidas, tais como a coluna lombar e sacral, os ombros e o pescoço. Esses fatores em seu conjunto apontam a necessidade urgente de modificação das tarefas nestes dois postos.

O rodízio de tarefas representa a medida emergencial mais adequada para minimizar os riscos de lesão do sistema osteomuscular dos trabalhadores. Entretanto, os organizadores do trabalho devem avaliar a possibilidade de redução do peso das caixas ou mesmo redefinir outros determinantes das tarefas que levem a uma modificação da freqüência de levantamentos e abaixamentos de caixas, o período de exposição às tarefas e um maior número de operadores.

Os resultados desta pesquisa podem justificar e subsidiar estudos futuros que venham a desenvolver tecnologias que minimizem os riscos de lesão do sistema osteomuscular dos trabalhadores que executam tarefas de MMC nas UB a fim de promoverlhes uma melhor qualidade de vida.

\section{AGRADECIMENTOS}

Os autores agradecem ao Conselho Nacional de Desenvolvimento Científico e Tecnológico (CNPq) e ao Fundo de Apoio Ao Ensino, à Pesquisa e à Extensão (FAEPEX), pelo apoio financeiro.

Ciência Rural, v.39, n.4, jul, 2009. 


\begin{tabular}{|c|c|c|c|c|c|c|}
\hline & UB1 & UB2 & UB3 & UB4 & UB5 & UB6 \\
\hline $\begin{array}{l}\text { Descarregamento } \\
\text { de Caminhö́es }\end{array}$ & $\frac{1}{30}$ & 용 & $\frac{2}{9}$ & $\frac{2}{2}$ & 욜 & $\frac{8}{80}$ \\
\hline $\begin{array}{l}\text { LPR } \\
\text { IL }\end{array}$ & $\begin{array}{c}12,2 \mathrm{~kg} \\
1,9\end{array}$ & $\begin{array}{c}3,2 \mathrm{~kg} \\
7,5\end{array}$ & $\begin{array}{c}3,2 \mathrm{~kg} \\
7,5\end{array}$ & $\begin{array}{c}12,7 \mathrm{~kg} \\
1,9\end{array}$ & $\begin{array}{c}3,9 \mathrm{~kg} \\
6,2\end{array}$ & $\begin{array}{c}3,9 \mathrm{~kg} \\
6,2\end{array}$ \\
\hline $\begin{array}{l}\text { Alimentação de } \\
\text { Máquina }\end{array}$ & $8=$ & $\frac{8}{2 y}$ & $e_{i}$ & 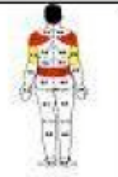 & 8 & 8 \\
\hline $\begin{array}{l}\text { LPR } \\
\text { IL }\end{array}$ & $\begin{array}{c}10,1 \mathrm{~kg} \\
2,3\end{array}$ & $\begin{array}{c}3,8 \mathrm{~kg} \\
6,0\end{array}$ & $\begin{array}{c}4,0 \mathrm{~kg} \\
6,0\end{array}$ & $\begin{array}{c}9,7 \mathrm{~kg} \\
2,4\end{array}$ & $\begin{array}{c}6,8 \mathrm{~kg} \\
3,5\end{array}$ & $\begin{array}{c}4,0 \mathrm{~kg} \\
6,0\end{array}$ \\
\hline Paletizaçäo 1 & & & & & & He \\
\hline $\begin{array}{c}\text { LPR } \\
\text { IL }\end{array}$ & & & & & & $\begin{array}{c}11,0 \mathrm{~kg} \\
2,0\end{array}$ \\
\hline Pesagem & $\frac{6}{8}$ & 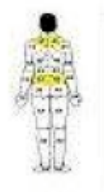 & $\frac{0}{4}$ & $\frac{0}{4 \cdot}$ & $\frac{\bullet}{9}$ & $\frac{2}{2}$ \\
\hline $\begin{array}{l}\mathrm{LPR} \\
\mathrm{IL}\end{array}$ & $\begin{array}{c}13,1 \mathrm{~kg} \\
1,6\end{array}$ & $\begin{array}{c}9,2 \mathrm{~kg} \\
1,8\end{array}$ & $\begin{array}{c}9,8 \mathrm{~kg} \\
2,2\end{array}$ & $\begin{array}{c}12,5 \mathrm{~kg} \\
1,7\end{array}$ & $\begin{array}{c}10,3 \mathrm{~kg} \\
2,1\end{array}$ & $\begin{array}{c}3,9 \mathrm{~kg} \\
5,6\end{array}$ \\
\hline Paletizaçǟo 2 & $\frac{8}{i 2}$ & 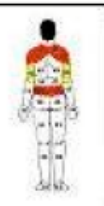 & $\therefore$ & $\frac{8}{40}$ & 2 & $\frac{2}{2}$ \\
\hline$\stackrel{\text { LPR }}{\mathrm{IL}}$ & $\begin{array}{c}12,5 \mathrm{~kg} \\
1,7\end{array}$ & $\begin{array}{c}8,9 \mathrm{~kg} \\
2,5\end{array}$ & $\begin{array}{c}9,3 \mathrm{~kg} \\
2,3\end{array}$ & $\begin{array}{c}12,1 \mathrm{~kg} \\
1,8\end{array}$ & $\begin{array}{c}9,3 \mathrm{~kg} \\
2,4\end{array}$ & $\begin{array}{c}4,2 \mathrm{~kg} \\
5,2\end{array}$ \\
\hline $\begin{array}{c}\text { Carregamento de } \\
\text { Caminhoües }\end{array}$ & $\dot{a}$ & $\stackrel{-}{a}$ & ${ }_{4}^{2} \frac{3}{4}$ & $\dot{0}$ & $\stackrel{\bullet}{-}$ & $\frac{1}{9}$ \\
\hline $\begin{array}{l}\text { LPR } \\
\text { IL }\end{array}$ & $\begin{array}{c}10,8 \mathrm{~kg} \\
2,0\end{array}$ & & & $\begin{array}{c}12,3 \mathrm{~kg} \\
1,8\end{array}$ & & \\
\hline
\end{tabular}

Figura 2 - Diagrama das áreas dolorosas e resultados de LPR e IL.

\section{REFERÊNCIAS}

BRASIL. Ministério do Trabalho e Emprego. Manual de aplicação da norma regulamentadora NR. 17. 2.ed. Brasília: MTE, 2002. Acesso em: 08 de Jan. 2006. On line. Disponível em: <http://www.mte.gov.br/seg_sau/pub_cne_manual_nr17.pdf>.
CHAFFIN, D.B. et al. Biomecânica ocupacional. 3.ed. Belo Horizonte: Ergo, 2001. 579p.

FERREIRA, M.D. Perdas na cadeia produtiva do tomate de mesa. In: WORKSHOP TOMATE NA UNICAMP, 2003, Campinas. Anais... Campinas-SP: FEAGRI, 2003. CD-Rom. 
FREIVALDS et al. A dynamic biomechanical evaluation of lifting maximum acceptable loads. Biomechanics, v.17, p.25162, 1984. Disponível em: http://www.sciencedirect.com/ science?_ob=ArticleURL\&_udi=B6T82-4C06GNX2 R\&_user $=687358 \&$ \&doc $=1 \&$ \& fmt $=\&$ \&orig $=$ search $\&$ _sort $=$ d \& view $=$ c \&_a c ct $=$ C $000037899 \&$ _versio $\mathrm{n}=1 \&$ u r l V e r s i o n $=0$ \&_u s e ri d $=687$ 358\&md5=2e267a5683a12165841089d3c9555096. Doi: $10.1016 / 0021-9290(84) 90136-2$.

GRANDJEAN, E. Manual de ergonomia: adaptando o trabalho ao homem. 4.ed. São Paulo: Artmed, 1998. 338p.

IIDA, I. Ergonomia. projeto e produção. 2.ed. São Paulo: Edgar Blucher, 2005. 614p.
TEIXEIRA, E.R. Sistematização de procedimentos necessários à aplicação da equação de levantamento do NIOSH: estudo descritivo da relação entre o índice de levantamento da equação revisada do NIOSH e a incidência de lombalgia numa amostra de trabalhadores. 2004. 239f. Dissertação (Mestrado em Engenharia mecânica) - Universidade Federal do Paraná, Curitiba, PR.

PEIXOTO, A. O mercado de tomate no Brasil e suas tendências. UNICAMP, 23 de maio de 2003. Disponível em: < http://www.feagri.unicamp.br/tomates/pdfs/wrktom001.pdf>.

WATERS, T.R. et al. Applications manual for revised NIOSH lifiting equation. 1994. Acesso em: 22 de jan. de 2006. On line. Disponível em: <http://www.cdc.gov/niosh/ docs/94-110/pdfs/94-110.pdf > . Acesso em: 24 de abr. de 2005. 